\title{
Symmetry and convergence properties for non-negative solutions of nonautonomous reaction-diffusion problems
}

\author{
Hess, Peter ; Poláčik, P
}

\begin{abstract}
Nonautonomous parabolic equations of the form $<$ jats:italic $>\mathrm{u}<$ jats:sub $>\mathrm{t}</$ jats:sub $></$ jats:italic $>-$ $\Delta<$ jats:italic $>\mathrm{u}</$ jats:italic $>=<$ jats:italic $>\mathrm{f}</$ jats:italic $>(<$ jats:italic $>\mathrm{u}, \mathrm{t}</$ jats:italic $>)$ on a symmetric domain are considered. Using the moving-hyperplane method, it is proved that any bounded nonnegative solution symmetrises as $<$ jats:italic $>t \rightarrow \infty</$ jats:italic $>$. This is then used to show that for nonlinearities periodic in $<$ jats:italic $>\mathrm{t}</$ jats:italic $>$, any non-negative bounded solution approaches a periodic solution.
\end{abstract}

DOI: https://doi.org/10.1017/s030821050002878x

Posted at the Zurich Open Repository and Archive, University of Zurich

ZORA URL: https://doi.org/10.5167/uzh-154020

Journal Article

Published Version

Originally published at:

Hess, Peter; Poláčik, P (1994). Symmetry and convergence properties for non-negative solutions of nonautonomous reaction-diffusion problems. Proceedings of the Royal Society of Edinburgh: Section A, 124(03):573-587.

DOI: https://doi.org/10.1017/s030821050002878x 


\title{
Symmetry and convergence properties for non-negative solutions of nonautonomous reaction-diffusion problems
}

\author{
Peter Hess $\uparrow$ \\ Mathematisches Institut, Universität Zürich, Rämistrasse 74, 8001 Zürich, \\ Switzerland \\ P. Poláčik \\ Institute of Applied Mathematics, Comenius University, Mlynská Dolina, \\ 82415 Bratislava, Slovakia \\ (MS received 22 January 1993. Revised MS received 26 April 1993)

\begin{abstract}
Nonautonomous parabolic equations of the form $u_{t}-\Delta u=f(u, t)$ on a symmetric domain negative solution symmetrises as $t \rightarrow \infty$. This is then used to show that for nonlinearities periodic in $t$, any non-negative bounded solution approaches a periodic solution.
\end{abstract} \\ are considered. Using the moving-hyperplane method, it is proved that any bounded non-
}

\section{Introduction}

In this paper we give an extension of the famous Gidas-Ni-Nirenberg result, on the symmetry of positive solutions of semilinear elliptic Dirichlet problems with symmetry [11], to nonautonomous parabolic equations. We consider the reactiondiffusion equation

$$
\left.\begin{array}{rlrl}
\partial_{t} u-\Delta u & =f(u, t) & & \text { in } \Omega \times(0, \infty), \\
u & =0 & & \text { on } \partial \Omega \times(0, \infty) .
\end{array}\right\}
$$

We assume $\Omega \subset \mathbb{R}^{N}(N \geqq 2)$ is a smooth bounded domain (suitably) symmetric with respect to the hyperplane $x_{1}=0$, and that $f$ satisfies suitable regularity conditions. Note that no further hypothesis on $f$ as a function of $t$, nor on $f(0, t)$, is imposed. Let $u: \bar{\Omega} \times[0, \infty) \rightarrow \mathbb{R}$ be a uniformly bounded non-negative (global) solution of $(*)$, with $u(\cdot, 0)=u_{0}$. Though equation $(*)$ is neither autonomous nor periodic in general, let us use the terminology " $\omega$-limit-set" for the set

$$
\tilde{\omega}\left(u_{0}\right):=\left\{z \in C_{0}^{1}(\bar{\Omega}): \exists t_{k}>\infty \text { such that } u\left(\cdot, t_{k}\right) \rightarrow z \text { in } C_{0}^{1}(\bar{\Omega}) \text { as } k \rightarrow \infty\right\} .
$$

By standard regularity theory, $\tilde{\omega}\left(u_{0}\right)$ is a nonempty compact subset of $E:=C_{0}^{1}(\bar{\Omega})$, and it consists here, of course, of non-negative functions. Our main result, Theorem 2.1, says that each element $z \in \tilde{\omega}\left(u_{0}\right)$ is symmetric with respect to $x_{1}=0$, and that either $z \equiv 0$ on $\bar{\Omega}$, or $z(x)>0$ for all $x \in \Omega$ and $\left(\partial z / \partial x_{1}\right)(x)<0$ for $x \in \Omega$ with $x_{1}>0$. The key tool in the proof of Theorem 2.1 is still the method of "moving hyperplanes"; we show that, roughly speaking, symmetry of $u(\cdot, t)$ "improves" as $t \rightarrow \infty$.

†Peter Hess died in 1992. 
In the autonomous case, $\tilde{\omega}\left(u_{0}\right)=\left\{u_{0}\right\}$ for any equilibrium solution $u_{0}$, and we get a slightly strengthened version of Gidas, $\mathrm{Ni}$ and Nirenberg's main results [11]. (Indeed, we do not have to assume that $u$ is positive in $\Omega$, at the cost of some additional condition on the normal to $\partial \Omega$ ). If we restrict $\Omega$ to be a ball, we thus also get the main result of Castro and Shivaji [5] which states that nontrivial nonnegative solutions are positive in $\Omega$. Observe that this assertion is not true in space dimension one, as the example $-u_{x x}=u-1$ in $x \in(-3 \pi, 3 \pi)$, having solution $u(x)=$ $\cos x+1$, shows. As a corollary (Corollary 2.9) of our main result, we obtain that any $T$-periodic non-negative solution of $(*)$ is symmetric in $x$, for all $t$ (indeed, $u(\cdot, t)$ always lies in the $\omega$-limit-set). We thus get another proof of a recent result of [10]. The same assertion holds for an almost periodic non-negative solution $u$ of $(*)$ (Corollary 2.10). This was pointed out to us by K. Schmitt.

We then use our main result to prove in Theorem 3.1 convergence of all bounded non-negative solutions of the $T$-periodic problem $(*)$ to $T$-periodic solutions of $(*)$. More precisely, we assume here that $\Omega=\mathbb{B}(0)$ is the unit ball in $\mathbb{R}^{N}$, that $f$ is smooth in $(u, t)$, and that the $T$-periodic non-negative solutions of $(*)$ are isolated (as fixed points of the associated Poincare map $S$ ). Any real-analytic, periodic function $f=$ $f(u, t)$ satisfying $f(0, \cdot) \geqq 0$, but $\not \equiv 0$, meets this assumption. We refer to Section 4 , also, for another sufficient condition; there also the case $f(0, \cdot) \equiv 0$ will be treated, in which the fixed points need no longer be isolated.

In the proof of Theorem 3.1 we consider the $\omega$-limit set

$$
\omega\left(u_{0}\right):=\left\{z \in C_{0}^{1}(\bar{\Omega}): \exists n_{k} \rightarrow \infty \text { such that } S^{n_{k}}\left(u_{0}\right) \rightarrow z \text { in } C_{0}^{1}(\bar{\Omega}) \text { as } k \rightarrow \infty\right\}
$$

of an initial condition $u_{0}$ which gives rise to a non-negative bounded solution of $(*)$. We show that $\omega\left(u_{0}\right)$ consists of a single element. This is done by studying the dynamics of $(*)$ on $\omega\left(u_{0}\right)$. A key observation is that, by Theorem 2.1, $\omega\left(u_{0}\right)$ consists of rotationally symmetric functions; thus the motion on $\omega\left(u_{0}\right)$ is described by an equation in one space dimension. Applying powerful one-dimensional tools, namely the properties of the zero number of the difference of solutions, we eventually obtain that $\omega\left(u_{0}\right)$ contains a stable fixed point (relative to $\left.\omega\left(u_{0}\right)\right)$. From this the convergence follows.

Theorem 3.1 is an extension of results in one space dimension $[4,7]$ to dimension $N \geqq 2$. For higher dimensions, only generic convergence to linearly or neutrally stable subharmonic solutions (of some period $n T, n \geqq 1$ ) is known in general $[17,18]$. For certain restricted classes of problems, generic convergence to T-periodic solutions is proved in [15] by a perturbation argument. Convergence of all bounded solutions to $T$-periodic solutions does not hold in general; indeed Takáć $[21,22]$ and Dancer and Hess [9] gave examples of semilinear $T$-periodic problems admitting linearly stable subharmonic solutions. It is known to be true, however, for some specific equations including the periodic logistic diffusion equation and equation of Fisher's type $[13,14,16]$.

\section{Asymptotic symmetry in nonautonomous problems}

We consider the nonautonomous semilinear boundary value problem

$$
\left.\begin{array}{rlrl}
\partial_{t} u-\Delta u & =f(u, t) & & \text { in } \Omega \times(0, \infty), \\
u & =0 & & \text { on } \partial \Omega \times(0, \infty)
\end{array}\right\}
$$


Here $\Omega$ is a bounded domain in $\mathbb{R}^{N}(N \geqq 2)$ with boundary $\partial \Omega$ of class $C^{2+\alpha}(0<\alpha \leqq 1)$. Let $\ell:=\sup \left\{x_{1}: x \in \Omega\right\}$, and set $\Omega_{\lambda}:=\left\{x \in \Omega: x_{1}>\lambda\right\}$. Further, let $\mathscr{P}_{\lambda}$ denote the reflection on the hyperplane $H_{\lambda}:=\left\{x \in \mathbb{R}^{N}: x_{1}=\lambda\right\}$. We assume:

(D0) $\Omega$ is symmetric with respect to the hyperplane $H_{0}$;

(D1) $\mathscr{P}_{\lambda}\left(\Omega_{\lambda}\right) \subset \Omega$ for all $\lambda \in(0, \ell)$;

(D2) for all $x \in \partial \Omega$ with $x_{1}>0$, the exterior normal vector $v(x)$ has component $v_{1}>0$.

Our hypotheses on the nonlinearity $f: \mathbb{R} \times \mathbb{R} \rightarrow \mathbb{R}$ are:

(F0) for each $M>0$ and $\tau \geqq 0, f(u, t)$ is Hölder-continuous of exponent $(\alpha,(\alpha / 2))$ in the region $[-M, M] \times[\tau, \tau+1]$, with Hölder-norm bounded by a constant independent of $\tau$;

(F1) for each $M>0, f(u, t)$ is uniformly Lipschitz-continuous in $u$ in the region $[-M, M] \times[0, \infty)$.

Let $u \in C^{2,1}(\bar{\Omega}+[0, \infty))$ be an $L^{\infty}$-bounded non-negative solution of (*). Then $u$ is globally defined, and the positive semiorbit $\gamma^{+}\left(u_{0}\right):=\{u(\cdot, t): t \geqq 0\}$ with $u_{0}:=u(\cdot, 0)$ is relatively compact in the space $E:=C_{0}^{1}(\bar{\Omega})$. The $\omega$-limit-set $\tilde{\omega}\left(u_{0}\right)$ is nonempty and compact in $E$. Let $\partial_{1}:=\partial / \partial x_{1}$.

THEOREM 2.1. Each $z \in \tilde{\omega}\left(u_{0}\right)$ is symmetric with respect to $H_{0}$, and either $z \equiv 0$ on $\bar{\Omega}$, or $z(x)>0$ for all $x \in \Omega$ and $\partial_{1} z(x)<0$ for $x \in \Omega$ with $x_{1}>0$.

Proof. This is accomplished in a sequence of steps. The outline is as follows. Given a non-negative solution $u(x, t)$, we define a number $\lambda^{*}(t)$, the limit for the process of moving hyperplanes, which, roughly speaking, measures the symmetry of $u(\cdot, t)$ in the direction $x_{1}$ and equals zero if and only if $u(\cdot, t)$ has the "full" symmetry. After a preparation (Step (i)), where we show that $\lambda^{*}(t)<\ell$ for small $t>0$, we prove that $\lambda^{*}(t)$ is decreasing, strictly unless $\lambda^{*}(t)=0$. As a variant of the invariance principle we then obtain that all limit points of $u$ must be symmetric.

We now give the details.

Step (i). Let $\varepsilon>0$ be given. Without loss of generality we may assume that $u \neq 0$ on $\Omega \times[0, \varepsilon)$ (otherwise $u(\cdot, t)=0$ for all $t$, and the conclusion of Theorem 2.1 is trivial).

Claim 2.2. There exists a nontrivial closed interval $I \subset(0, \varepsilon)$ such that for each $t \in I$ and all $\lambda<\ell$ sufficiently close to $\ell$ (not depending on $t$ ), it is:

$$
\begin{cases}u\left(\mathscr{P}_{\lambda} x, t\right)>u(x, t) & \text { for all } x \in \Omega_{\lambda}, \\ \partial_{1} u(x, t)<0 & \text { for all } x \in H_{\lambda} \cap \Omega .\end{cases}
$$

Proof. Let $I \subset(0, \varepsilon)$ be a nontrivial closed interval such that $f(0, t) \equiv 0$ for all $t \in I$, or $f(0, t)>0$ for all $t \in I$, or $f(0, t)<0$ for all $t \in I$. Let $\underline{x} \in \partial \Omega$ with $\underline{x}_{1}=\ell$. We show that for each $t \in I$, either $\partial_{1} u(\underline{x}, t)<0$, or $\partial_{1} u(\underline{x}, t)=0$ and $\partial_{1}^{2} u(\underline{x}, t)>0$. This then implies, by a compactness argument, that $\partial_{1} u(x, t)<0$ for all $t \in I$ and $x \in \Omega$ sufficiently near $x$. The claim now follows easily.

Consider first the case $f(0, \cdot) \equiv 0$ on $I$. Then $u$ satisfies the linear equation

$$
\partial_{t} u-\Delta u=a(x, t) u \quad \text { in } \Omega \times I,
$$

where $a: a(x, t)=\int_{0}^{1} \partial_{u} f(s u(x, t), t) d s$ is bounded on $\bar{\Omega} \times I$. For $t \in I, u$ attains its 
minimum value 0 at $(\underline{x}, t)$. Since $u$ is nontrivial, the parabolic maximum principle implies that $\partial_{1} u(\underline{x}, t)<0$.

In case $f(0, \cdot)>0$ on $I$, assume to the contrary that there exists $t_{0} \in I$ such that both $\partial_{1} u\left(x, t_{0}\right)=0$ and $\partial_{1}^{2} u\left(\underline{x}, t_{0}\right)=0$. Since $u\left(\cdot, t_{0}\right)$ vanishes on $\partial \Omega$, it follows that $\Delta u\left(\underline{x}, t_{0}\right)=0$. Hence, by the equation $(*), \partial_{t} u\left(x, t_{0}\right)=f\left(0, t_{0}\right)>0$, which implies that $u(x, t) \neq 0$ for $t \neq t_{0}, t$ close to $t_{0}$, a contradiction to the boundary conditions.

The case $f(0, \cdot)<0$ on $I$ is treated similarly.

Step (ii). Let $I$ be as above, let $\underline{t} \in I$, and take $t \geqq \underline{t}$. We define

$$
\lambda^{*}(t):=\inf \left\{\lambda>0: u\left(\mathscr{P}_{\lambda} x, t\right) \geqq u(x, t) \text { and } \partial_{1} u(x, t) \leqq 0 \text { for all } x \in \Omega \text { with } x_{1} \geqq \lambda\right\} .
$$

ClaIm 2.3. $\lambda^{*}(t)$ is decreasing in t, strictly unless $\lambda^{*}(\underline{t})=0$.

Proof. Let $\bar{t}>\underline{t}$ be arbitrary. By Step (i) we know that $\lambda^{*}(\underline{t})<\ell$. Take any $\lambda \in\left[\lambda^{*}(t), \ell\right)$. We know in particular that

$$
\begin{cases}u\left(\mathscr{P}_{\lambda} x, \underline{t}\right) \geqq u(x, \underline{t}) & \text { for all } x \in \Omega_{\lambda}, \\ \partial_{1} u(x, t) \leqq 0 & \text { for all } x \in H_{\lambda} \cap \Omega .\end{cases}
$$

Consider the function $w_{\lambda}: w_{\lambda}(x, t)=u\left(\mathscr{P}_{\lambda} x, t\right)-u(x, t)$. Since $u\left(\mathscr{P}_{\lambda} x, t\right)$ is also a solution of $(*)$ (disregarding boundary conditions) for $x \in \Omega_{\lambda}$, we see that $w_{\lambda}$ satisfies a linear equation

$$
\partial_{t} w_{\lambda}-\Delta w_{\lambda}=c(x, t) w_{\lambda}
$$

in $Q_{\lambda}:=\Omega_{\lambda} \times[\underline{t}, \bar{t}]$, with

$$
c(x, t)=\int_{0}^{1} \partial_{u} f\left(u(x, t)+s w_{\lambda}(x, t), t\right) d s
$$

and $w_{\lambda} \geqq 0$ on the parabolic boundary $\left(\Omega_{\lambda} \times\{\underline{t}\}\right) \cup\left(\partial \Omega_{\lambda} \times[\underline{t}, \bar{t}]\right)$ of $Q_{\lambda}$. By the maximum principle, $w_{\lambda} \geqq 0$ in $Q_{\lambda}$. In particular, $w_{\lambda}(\cdot, \bar{t}) \geqq 0$ in $\Omega_{\lambda}$ and (since $w_{\lambda}=0$ on $\left.H_{\lambda} \cap \Omega\right) \partial_{1} w_{\lambda}(x, \bar{t}) \geqq 0$ for $x \in H_{\lambda} \cap \Omega$. By the definition of $w_{\lambda}$, the latter implies that $\partial_{1} u(x, \bar{t}) \leqq 0$ for $x \in H_{\lambda} \cap \Omega$. It follows that $\lambda^{*}(\bar{t}) \leqq \lambda$, and then $\lambda^{*}(\bar{t}) \leqq \lambda^{*}(\underline{t})$. in $t$.

The same argument applies to general $\underline{t}<\bar{t}$ and proves that $\lambda^{*}(t)$ is nonincreasing

Now assume $\lambda^{*}(t)>0$. To show that $\lambda^{*}(t)$ is strictly decreasing, we apply the above argument with $\lambda=\lambda^{*}(\underline{t})$ using the full force of the maximum principle. Indeed, either

(a) $w_{\lambda}(\cdot, t) \gg 0$ for $\underline{t}<t \leqq \underline{t}$

(which means that $w_{\lambda}(x, t)>0$ for all $x \in \Omega_{\lambda}$, and at $x \in \partial \Omega_{\lambda}$ either $w_{\lambda}(x, t)>0$ or $w_{\lambda}(x, t)=0$ with negative exterior normal derivative where the normal to $\partial \Omega_{\lambda}$ is defined), or

(b) $w_{\lambda}=0$ in $Q_{\lambda}$.

In view of the assumption $\lambda^{*}(\underline{t})>0$, the claim now follows from the next two lemmas.

LemMa 2.4. In case (a), $\lambda^{*}(\bar{t})<\lambda=\lambda^{*}(\underline{t})$.

Proof. For all $x \in H_{\lambda} \cap \Omega$ and $t \in(\underline{t}, t], \partial_{1} w_{\lambda}(x, t)>0$ and thus $\partial_{1} u(x, t)<0$. Let $I \subset(\underline{t}, \hat{t})$ be a nontrivial closed interval as in Step (i). By the same argument, in any point $\underline{x} \in H_{\lambda} \cap \partial \Omega$, with exterior normal $v$ to $\Omega$ (having $x_{1}$-component $v_{1}>0$ ) and 
for any $t \in I$ we have either

$\partial_{v} u(\underline{x}, t)<0 \quad$ (whence $\partial_{1} u(\underline{x}, t)<0$ since all tangential derivatives vanish),

or

$$
\left.\partial_{v} u(\underline{x}, t)=0 \quad \text { and } \quad \partial_{v}^{2} u(\underline{x}, t)>0 \quad \text { (whence } \partial_{1} u(\underline{x}, t)=0 \text { and } \partial_{1}^{2} u(\underline{x}, t)>0\right) .
$$

We infer that $\partial_{1} u(x, t)<0$ for all $x \in \bar{\Omega}$ with $x_{1}<\lambda$ close to $\lambda$ (depending on $t \in I$ ). Thus $\lambda$ can be properly decreased for each $t \in I$, such as to still satisfy the inequalities in (2.1). We thus obtain

$$
\lambda^{*}(\bar{t}) \leqq \lambda^{*}(t)<\lambda=\lambda^{*}(\underline{t})
$$

LEMMA 2.5. In case $(\mathrm{b}), \lambda^{*}(\underline{t})=0$.

Proof. Assume, to the contrary, that $\lambda=\lambda^{*}(\underline{t})>0$. Hence $u(\cdot, \underline{t}) \neq \equiv$ in $\Omega$. Since $w_{\lambda} \equiv 0$, we have

$$
u\left(\mathscr{P}_{\lambda} x, t\right)=u(x, t) \quad \text { for all }(x, t) \in Q_{\lambda} .
$$

Let $\underline{x} \in H_{\lambda} \cap \partial \Omega$. In this point

$$
\partial_{t} u(\underline{x}, t)-\Delta u(\underline{x}, t)=f(0, t) \text { for } t \in[\underline{t}, \underline{t}] .
$$

By (2.2), it follows that $\partial_{1} u(x, t)=0$, first in $x \in H_{\lambda} \cap \Omega$ and then by passage to the limit $x \rightarrow \underline{x}$ in $\underline{x}$. Since $v_{1}>0$ and all the tangential derivatives of $u$ at $\underline{x}$ vanish, we get

$$
\operatorname{grad} u(\underline{x}, t)=0, \text { for all } t \in[t, \bar{t}] \text {. }
$$

Moreover, again by (2.2) and the knowledge on $\partial_{1} u$, we have $\partial_{1}^{2} u(x, t) \leqq 0$, which implies that $\partial_{v}^{2} u(x, t) \leqq 0$ for all $t \in[t, \bar{t}]$. Since $u$ is non-negative and satisfies Dirichlet boundary conditions, by (2.4) the only possibility is that $\partial_{v}^{2} u(\underline{x}, t)=0$. Hence $\Delta u(x, t)=0$ for all $t \in[\underline{t}, \bar{t}]$. Also $\partial_{t} u(x, t)=0$ for all $t$. Thus, by the equation (2.3), $f(0, t)=0$ for all $t \in[\underline{t}, t]$. Now the maximum principle asserts that $\partial_{v} u(\underline{x}, t)<0$ (since $u(\cdot, t)$ is nontrivial), contradicting (2.4). It follows that $\lambda=\lambda^{*}(t)=0$.

Step (iii). Since $\lambda^{*}(t)$ is monotone decreasing, there exists

$$
\lambda_{\infty}:=\lim _{t \rightarrow \infty} \lambda^{*}(t)
$$

Of course, $0 \leqq \lambda_{\infty}<\ell$.

Claim 2.6. If $z \in \tilde{\omega}\left(u_{0}\right)$, then

$$
\left\{\begin{array}{l}
z\left(\mathscr{P}_{\lambda_{\infty}} x\right) \geqq z(x) \\
\partial_{1} z(x) \leqq 0
\end{array} \text { for all } x \in \Omega \text { with } x_{1} \geqq \lambda_{\infty} .\right.
$$

Proof. Let $t_{k} \rightarrow \infty$ be such that $u\left(\cdot, t_{k}\right) \rightarrow z$ in $E$, and let $\lambda>\lambda_{\infty}$ be arbitrary. Then $\lambda>\lambda^{*}\left(t_{k}\right)$ for $k \geqq k_{\lambda}$, and hence

$$
\left\{\begin{array}{l}
u\left(\mathscr{P}_{\lambda} x, t_{k}\right) \geqq u\left(x, t_{k}\right) \\
\partial_{1} u\left(x, t_{k}\right) \leqq 0
\end{array} \quad \forall x \in \Omega: x_{1} \geqq \lambda, \quad \forall k \geqq k_{\lambda} .\right.
$$

Passage to the limit $k \rightarrow \infty$ yields the result with $\lambda_{\infty}$ replaced by $\lambda$. Since this holds for all $\lambda>\lambda_{\infty}$, the claim follows by continuity. 
Step (iv). Let $z \in \tilde{w}\left(u_{0}\right)$.

ClaIM 2.7. $z$ is symmetric with respect to the hyperplane $H_{0}$.

Proof. We assume $z \neq \equiv$. Let $t_{k} \rightarrow \infty: u\left(\cdot, t_{k}\right) \rightarrow z$ in $E$. Thus $u\left(\cdot, t_{k}\right) \not \equiv 0$ for large $k$.

We set

$$
u_{k}(x, t):=u\left(x, t+t_{k}-1\right) \quad(0 \leqq t \leqq 1)
$$

and

$$
f_{k}(u, t):=f\left(u, t+t_{k}-1\right)
$$

Then

$$
\begin{cases}\partial_{t} u_{k}-\Delta u_{k}=f_{k}\left(u_{k}, t\right) & \text { in } \Omega \times[0,1], \\ u_{k}=0 & \text { on } \partial \Omega \times[0,1] .\end{cases}
$$

Let $M:=\sup \left\{\|u(\cdot, t)\|_{L^{\infty}}: t \geqq 0\right\}$. We remark that the sequence $\left(f_{k}\right)_{k \in \mathbb{N}}$ is relatively compact in $C^{\beta, \beta / 2}([-M, M] \times[0,1])$ for $0<\beta<\alpha$ by (F0). Thus (for a subsequence) $f_{k} \rightarrow \tilde{f}$ in $C^{\beta, \beta / 2}([-M, M] \times[0,1])$, and $\tilde{f}$ is Lipschitz continuous in $u$ in $[-M, M] \times[0,1]$ by $(\mathrm{F} 1)$. By standard a priori estimates, the sequence $\left(u_{k}\right)_{k \in \mathbb{N}}$ is relatively compact in $C^{1+\beta,(1+\beta) / 2}(\bar{\Omega} \times[0,1])$, and hence (for a further subsequence) $u_{k} \rightarrow \tilde{u}$, where $\tilde{u}$ is a classical solution of

$$
\begin{cases}\partial_{t} \tilde{u}-\Delta \tilde{u}=\tilde{f}(\tilde{u}, t) & \text { in } \Omega \times[0,1], \\ \tilde{u}=0 & \text { on } \partial \Omega \times[0,1] .\end{cases}
$$

We note that $\tilde{u}(\cdot, t) \in \tilde{\omega}\left(u_{0}\right)$ for each $t \in[0,1]$; in particular $\tilde{u}(\cdot, 1)=z$.

For $\lambda \geqq 0$ we also define $w_{\lambda, k}$ :

$$
w_{\lambda, k}(x, t)=u_{k}\left(\mathscr{P}_{\lambda} x, t\right)-u_{k}(x, t) .
$$

Then $w_{\lambda, k} \rightarrow \tilde{w}_{\lambda}$ in $C^{1+\beta,(1+\beta) / 2}\left(\bar{\Omega}_{\lambda} \times[0,1]\right)$, where $\tilde{w}_{\lambda}(x, t)=\tilde{u}\left(\mathscr{P}_{\lambda} x, t\right)-\tilde{u}(x, t)$. For $\lambda \in\left[\lambda^{*}\left(t_{k}-1\right), \ell\right)$, we have

$$
\left\{\begin{array}{l}
\partial_{t} w_{\lambda, k}-\Delta w_{\lambda, k}=c_{\lambda, k} w_{\lambda, k} \\
w_{\lambda, k} \geqq 0
\end{array} \text { in } \tilde{Q}_{\lambda}:=\Omega_{\lambda} \times[0,1]\right.
$$

By passage to the limit $k \rightarrow \infty$, we obtain

$$
\left\{\begin{array}{l}
\partial_{t} \tilde{w}_{\lambda}-\Delta \tilde{w}_{\lambda}=\tilde{c}_{\lambda} \tilde{w}_{\lambda} \\
\tilde{w}_{\lambda} \geqq 0
\end{array} \text { in } \tilde{Q}_{\lambda} .\right.
$$

(2.7) holds for all $\lambda>\lambda_{\infty}$, and by passage to the limit $\lambda \downarrow \lambda_{\infty}$ also for $\lambda=\lambda_{\infty}$, with a bounded $\tilde{c}_{\lambda_{\infty}}$.

Now assume $\lambda_{\infty}>0$. By the maximum principle, applied to (2.7) with $\lambda=\lambda_{\infty}$, we have either

(a) $\tilde{w}_{\lambda_{\infty}}(\cdot, t) \gg 0$ for $0<t \leqq 1$, or

(b) $\tilde{w}_{\lambda_{\infty}} \equiv 0$ in $\tilde{Q}_{\lambda_{\infty}}$.

In case (a), it follows that $w_{\lambda_{\infty}, k}(\cdot, t) \gg 0$ for large $k$ (in the sense described in case (a) of Step (ii)). As in Lemma 2.4, we see that $\lambda^{*}\left(t_{k}\right)<\lambda_{\infty}$, contradicting the monotonicity of $\lambda^{*}(t)$ and the definition of $\lambda_{\infty}$. In the case (b), we get a contradiction to $\lambda_{\infty}>0$ as in Lemma 2.5 . 
Hence $\lambda_{\infty}=0$, and (2.5) holds (with $\lambda_{\infty}=0$ ). Using also the symmetric argument starting at $\lambda=-\ell$, we infer that $z$ is symmetric with respect to the hyperplane $H_{0}$.

Step (v). Assume that $z \in \tilde{w}\left(u_{0}\right)$ does not vanish identically.

ClaIm 2.8. $z(x)>0$ for all $x \in \Omega$, and $\partial_{1} z(x)<0$ for $x \in \Omega$ with $x_{1}>0$.

Proof. Assume there is $\tilde{x} \in \Omega$ with $\tilde{x}_{1}>0$ such that $\partial_{1} z(\tilde{x}) \geqq 0$ (this is true in particular if $z(\tilde{x})=0$ ). Set $\lambda=\tilde{x}_{1}$, and apply the arguments of Step (iv). In case $\tilde{w}_{\lambda}(\cdot, t) \gg 0$ for $0<t \leqq 1$ (i..e case (a)), we get $\partial_{1} \tilde{w}_{\lambda}(\tilde{x}, 1)^{\prime}>0$ by the maximum principle, and hence (since $z=\tilde{u}(\cdot, 1)) \partial_{1} z(\tilde{x})<0$, a contradiction. In case $\tilde{w}_{\lambda} \equiv 0$, as in Lemma 2.5 we obtain $\tilde{f}(0, t)=0$ for all $t \in[0,1]$, and the maximum principle applied to (2.6) implies that $\tilde{u}(x, t)>0$ for all $x \in \Omega, 0<t \leqq 1$, since $z=\tilde{u}(\cdot, 1) \neq \equiv$. Looking e.g. at a point $\underline{x} \in \partial \Omega$ with $\underline{x}_{1}=\ell$, we then conclude that $\tilde{w}_{\lambda}(\underline{x}, t)>0$ for $0<t \leqq 1$, contradicting the assumption that $\tilde{w}_{\lambda} \equiv 0$.

Hence $\partial_{1} z(x)<0$ for all $x \in \Omega$ with $x_{1}>0$, which implies that $z(x)>0$ in $\Omega$.

In these five steps we have proved Theorem 2.1 completely.

We get the immediate corollaries:

COROLlaRY 2.9. Let $u \in C^{2,1}(\bar{\Omega} \times \mathbb{R})$ be a non-negative T-periodic solution of (*) for some $T>0$. Then, for each $t, u(\cdot, t)$ is symmetric with respect to $H_{0}$, and either $u(\cdot, t) \equiv 0$ in $\Omega$, or $u(x, t)>0$ for any $x \in \Omega$ and $\partial_{1} u(x, t)<0$ for $x \in \Omega$ with $x_{1}>0$.

This is a slight extension of the main result of $[10]$, proved there by quite different arguments and with fewer regularity assumptions on $\partial \Omega$ and $u$.

COROLlaRY 2.10. Let $u \in C^{2,1}(\bar{\Omega} \times \mathbb{R})$ be a non-negative solution of (*) almost periodic in $t$ uniformly for $x \in \bar{\Omega}$. Then the assertions of Corollary 2.9 hold.

Proof. Let $t_{n} \rightarrow \infty$ be an arbitrary sequence. By almost-periodicity, there exists a subsequence $\left(t_{n_{k}}\right)$ such that $u\left(\cdot, t+t_{n_{k}}\right) \rightarrow v(\cdot, t)$, uniformly on $\bar{\Omega} \times \mathbb{R}$. Obviously, for each $t, v(\cdot, t)$ is an element of $\tilde{\omega}\left(u_{0}\right)$, hence $v$ is symmetric in $x_{1}$ by Theorem 2.1. Now $v$ lies in the hull of $u$, which implies also that $u$ is an element of the hull of $v$. But symmetry (in $x_{1}$ ) of $v$ implies symmetry of the elements in the hull of $v$.

We thank K. Schmitt who kindly pointed out Corollary 2.10 (inclusive proof) to us.

\section{Convergence in periodic problems}

We consider again problem

$$
\left.\begin{array}{rlrl}
\partial_{t} u-\Delta u & =f(u, t) & & \text { in } \Omega \times(0, \infty), \\
u & =0 & & \text { on } \partial \Omega \times(0, \infty),
\end{array}\right\}
$$

assuming that $\Omega=\mathbb{B}(0)$ is the open unit ball in $\mathbb{R}^{N}$, and that

(F2) $f$ is of class $C^{\infty}$ in $(u, t)$ and $T$-periodic in $t$.

Let $S: E=C_{0}^{1}(\bar{\Omega}) \supset \operatorname{dom}(S) \rightarrow E: u(\cdot, 0) \mapsto u(\cdot, T)$ be the Poincaré operator associated with (*). Of course $q \in \operatorname{dom}(S)$ is a fixed point of $S$ if and only if $q$ is the initial value of a $T$-periodic solution of $(*)$. We moreover assume in this section that

(P) the fixed points of $S$ giving rise to non-negative periodic solutions of $(*)$ are isolated in $E$. 
Sufficient conditions implying (P) will be given in Section 4 (Propositions 4.2-4.3); there we consider also a case where $(\mathrm{P})$ need not hold.

THEOREM 3.1. Let $\Omega$ be a ball, let $(\mathrm{F} 2)$ and $(\mathrm{P})$ be satisfied, and let $u$ be a non-negative, uniformly bounded $C^{2,1}$-solution of $(*)$. Then $u(\cdot, t)$ converges, as $t \rightarrow \infty$, to a T-periodic solution $w(\cdot, t)$ of $(*)$ in $E$.

By Theorem 2.1, the limit solution $w$ is rotationally symmetric in $x \in \Omega$.

Let $E_{\mathrm{rot}}:=\{z \in E: z$ is rotationally symmetric $\}$. We shall identify $E_{\text {rot }}$ with the subspace of $C^{1}([0,1])$ of functions $z(r)(r=\|x\|)$ satisfying the boundary conditions $z_{r}(0)=z(1)=0$. By the $S^{1}$-symmetry of the equation, the restriction $S_{\text {rot }}$ of the Poincaré operator $S$ to the domain $E_{\text {rot }}$ maps into $E_{\text {rot }}$ and is the Poincaré map of the reduced problem

$$
\left.\begin{array}{cl}
\partial_{t} u-u_{r r}-\frac{N-1}{r} u_{r}=f(u, t) & \text { in }(0,1) \times(0, \infty), \\
u_{r}(0, t)=u(1, t)=0 & (t>0) .
\end{array}\right\}
$$

Let $\omega\left(u_{0}\right):=\left\{z \in E: \exists n_{k} \rightarrow \infty\right.$ such that $S^{n_{k}}\left(u_{0}\right) \rightarrow z$ in $E$ as $\left.k \rightarrow \infty\right\}$ be the $\omega$-limit set of $u_{0} \in E$ associated to the periodic problem (*); it is nonempty and compact if the solution $u$ with initial value $u(\cdot, 0)=u_{0}$ is bounded in $L^{\infty}$. For non-negative solutions of $(*), \omega\left(u_{0}\right) \subset E_{\text {rot }}$ by Theorem 2.1. The assertion of Theorem 3.1 is that for nonnegative solutions $\omega\left(u_{0}\right)=\{q\}$ for some $q$. Then $q$ is necessarily a fixed point of $S$, and hence also of $S_{\text {rot }}$. In order to prove that $\omega\left(u_{0}\right)$ is a singleton, we study the dynamics of $\left(*_{\text {rot }}\right)$. We use results on the evolution of the zero number along global solutions $v$ of linear homogeneous equations of the form

$$
\left.\begin{array}{cl}
\partial_{t} v-v_{r r}-\frac{N-1}{r} v_{r}=c(r, t) v & \text { in }(0,1) \times(0, \infty), \\
v_{r}(0, t)=v(1, t)=0 & (t>0) .
\end{array}\right\}
$$

with smooth $c$. Observe that by (F2),the difference $v=u-\bar{u}$ of any two solutions $u, \bar{u}$ of $\left(*_{\text {rot }}\right)$ satisfies such an equation.

For a solution $v(r, t)$ of $\left({ }^{*}{ }_{\operatorname{lin}}\right)$, let $\zeta(v(\cdot, t))$ denote the number (possibly infinite) of zeros of the function $r \mapsto v(r, t)$ in the interval $r \in[0,1]$. In the following proposition, we collect some properties of $\zeta(v(\cdot, t))$ and their basic consequences for the equation $\left({ }_{\text {rot }}\right)$.

Proposition 3.2. (A) Nonincrease of the zero number. Let $u$ and $\bar{u}$ be global solutions of $\left(*_{\mathrm{rot}}\right)$, and set $v:=u-\bar{u}$. Then

(i) $\zeta(v(\cdot, t))<\infty$ for all $t>0$;

(ii) $\zeta(v(\cdot, t))$ is nonincreasing in $t ;$

(iii) if for some $t=t_{0}>0, v\left(\cdot, t_{0}\right)$ has a zero of multiplicity higher than one in $[0,1]$, then $\zeta(v(\cdot, t))$ drops strictly at $t=t_{0}$.

(B) Convergence. (i) If $u_{0} \in E_{\text {rot }}$ has a bounded semiorbit for $t>0$, the solution of $\left({ }^{*}{ }_{\text {rot }}\right)$ converges as $t \rightarrow \infty$ to a T-periodic solution, i.e. $\omega\left(u_{0}\right)=\{q\}$ with $S_{\text {rot }}(q)=q$.

(ii) If $u_{0}$ has a relatively compact negative orbit in $E_{\text {rot }}$, the $\alpha$-limit-set $\alpha\left(u_{0}\right)$ consists 
of a single fixed point of $S_{\mathrm{rot}}$. (Note that a negative orbit is uniquely defined by backward uniqueness.)

(C) The linearised Poincaré map. Let $e \in E_{\text {rot }}$ be a fixed point of $S_{\text {rot. }}$ Then the spectrum $\sigma\left(d S_{\mathrm{rot}}(e)\right) \backslash\{0\}$ consists of a sequence of simple real eigenvalues $\lambda_{1}>$ $\lambda_{2}>\ldots>0$; the associated $n$-th eigenfunction $\varphi_{n}(r)$ has only simple zeros in $[0,1]$, and $\zeta\left(\varphi_{n}\right)=n$.

Proof. These results are extensions to $\left(*_{\text {rot }}\right)$ of well-known results on one-dimensional problems without the singularity at $r=0$. In the present case, $(A)$ is an immediate consequence of the results in [1, Sections 3,4]. Assertions (B) and (C) follow from (A) just as in the case of nonsingular equations. See $[4,7]$ for (B)(i) and $[6$, Section 2] for the proof of (C). Property (B)(ii) does not seem to have been stated in the existing literature (its proof will be contained in a forthcoming paper of the second author and X.-Y. Chen). Under the assumption (P), however, (B)(ii) is a consequence of the fact (proved for nonsingular equations in [6, Lemma 4.6]) that the set of nonwandering points of $F_{\text {rot }}$ consists of fixed points. In particular, any $\alpha$-limit set must consist of fixed points, hence must be a continuum of fixed points. Condition (P) then implies that it is a single point.

REMARK 3.3. In this section the only reason for the assumption that $f$ is $C^{\infty}$, is that we need $c$ in $\left(*_{\text {lin }}\right)$ to be smooth in order to be able to apply the results of [1, Sections 3, 4]. Most probably, this regularity assumption can be significantly relaxed.

We now turn to the next proof.

Proof of Theorem 3.1. Let us write $K:=\omega\left(u_{0}\right)$ in the sequel; we know that $K \subset E_{\text {rot }}$ is compact, and that (by backward uniqueness)

$$
h:=\left.S_{\text {rot }}\right|_{K}: K \rightarrow K
$$

is a homeomorphism. By Proposition 3.2(B), $K$ can only consist of fixed points of $S_{\text {rot }}$ and of connecting heteroclinic and homoclinic orbits. Since $K$ is compact, by (P) there are only finitely many fixed points in $K$. We show in two steps that $K$ does not contain cycles (including homoclinic orbits)

$$
e_{0} \leadsto e_{1} \leadsto \ldots m e_{p} \leadsto e_{0} \quad(p \in \mathbb{N})
$$

Here $e_{j}(0 \leqq j \leqq p)$ are pairwise distinct fixed points and the notation $e_{j} \leadsto e_{j+1}$ means that there is an entire orbit $\left(h^{n}(y)\right)_{n \in \mathbb{Z}}$ in $K \backslash\left\{e_{j}, e_{j+1}\right\}$ with $h^{-n}(y) \rightarrow e_{j}$, $h^{n}(y) \rightarrow e_{j+1}$ as $n \rightarrow \infty$. In the third step we then show the existence of a stable fixed point $\hat{e}$ of $h$, which then implies that $K=\{\hat{e}\}$.

Step (i). We have

CLAIM 3.4. $K$ does not contain any cycle $e_{0} \leadsto e_{1} \leadsto \ldots \leadsto e_{p} \leadsto e_{0}, p \geqq 1$.

This follows from the following two lemmas:

LemMa 3.5. Let $e_{0}, e_{1}$ be fixed points of $S_{\text {rot }}, e_{0} \neq e_{1}$. Then $e_{0}-e_{1} \in C^{1}([0,1])$ has only simple zeros in $[0,1]$.

Proof. Proposition 3.2(A) and the periodicity of $u\left(r, t ; e_{0}\right)-u\left(r, t ; e_{1}\right)$ in $t$ immediately imply the claim. (Here, $u\left(\cdot, \cdot ; u_{0}\right)$ denotes the solution of $(*$ rot $)$ having value $u_{0}$ at $t=0$.) 
LeMMA 3.6. Let $e_{0}, e_{1}, e_{2}$ be distinct fixed points of $S_{\mathrm{rot}}$, with connecting orbits $e_{0} \leadsto e_{1} \leadsto e_{2}$. Then

$$
\zeta\left(e_{2}-e_{1}\right) \leqq \zeta\left(e_{1}-e_{0}\right)
$$

If equality holds in (3.1), then at $r=0$,

$$
\left(e_{2}(0)-e_{1}(0)\right)\left(e_{1}(0)-e_{0}(0)\right)>0 .
$$

Proof. There exist solutions $u^{0}(r, t)$ and $u^{1}(r, t)$ of $\left({ }^{*}\right.$ rot $)$ on $[0,1] \times \mathbb{R}$ associated with the connecting orbits, i.e. solutions with

$$
\begin{aligned}
& u^{i}(\cdot, n T) \rightarrow e_{i} \quad(n \rightarrow-\infty) \\
& u^{i}(\cdot, n T) \rightarrow e_{i+1} \quad(n \rightarrow \infty)
\end{aligned} \text { in } E_{\mathrm{rot}}
$$

$(i=0,1)$. Let $v(r, t):=u^{1}(r, t)-u^{0}(r, t)$. By Lemma 3.5 and convergence in $C^{1}([0,1])$,

$$
\begin{array}{ll}
\zeta(v(\cdot, t))=\zeta\left(e_{1}-e_{0}\right) & \text { for } t=-n T \ll 1, \\
\zeta(v(\cdot, t))=\zeta\left(e_{2}-e_{1}\right) & \text { for } t=n T \gg 1 .
\end{array}
$$

Since $\zeta(v(\cdot, t))$ is nonincreasing in $t$, we get (3.1). If there is equality in $(3.1), v(\cdot, t)$ has only simple zeros in $[0,1]$ by Proposition 3.2(A). Since $v_{r}(0, t)=0$ for all $t$, it follows that $v(0, t) \neq 0$ for all $t$. We infer by the convergence of $v$ as $t \rightarrow \pm \infty$ that

$$
\left(e_{2}(0)-e_{1}(0)\right)\left(e_{1}(0)-e_{0}(0)\right) \geqq 0 .
$$

Equality in (3.2) is not possible by the boundary condition at $r=0$ and Lemma 3.5 .

It is now readily seen that Claim 3.4 holds, since for a cycle (with $p \geqq 1$ ) the numbers $\zeta\left(e_{j+1}-e_{j}\right)$ would all be equal for $0 \leqq j \leqq p$ by (3.1), while the strict inequalities

$$
\left(e_{j+2}(0)-e_{j+1}(0)\right)\left(e_{j+1}(0)-e_{j}(0)\right)>0
$$

for $0 \leqq j \leqq p-1$ would contradict that $e_{p+1}=e_{0}$.

Step (ii). We have

CLAIM 3.7. $K$ does not contain a homoclinic orbit $e m e$.

Proof. Assume there is a homoclinic oribit $\left(u_{n}\right)_{n \in \mathbb{Z}}$ in $K$ with $u_{n} \rightarrow e(n \rightarrow \pm \infty)$. There exists the associated solution $u(\cdot, t)$ of $\left({ }^{*}{ }_{\text {rot }}\right)$ on $t \in \mathbb{R}$, with $u(\cdot, n T)=u_{n}(n \in \mathbb{Z})$. Let $v(\cdot, t):=u(\cdot, t+T)-u(\cdot, t)$, and set $v_{n}:=v(\cdot, n T)=u_{n+1}-u_{n}$. Note that $\left\|v_{n}\right\| \rightarrow 0$ as $n \rightarrow \infty\left(\right.$ norm in $\left.E_{\text {rot }}\right)$. We have

$$
v_{n+1}=u_{n+2}-u_{n-1}=S_{\text {rot }}\left(u_{n+1}\right)-S_{\text {rot }}\left(n_{n}\right)=d S_{\text {rot }}(e) v_{n}+\xi(n),
$$

where $\xi(n)=\int_{0}^{1}\left[d S_{\text {rot }}\left(u_{n}+s v_{n}\right)-d S_{\text {rot }}(e)\right] v_{n} d s$ satisfies

$$
\lim _{n \rightarrow \pm \infty} \frac{\xi(n)}{\left\|v_{n}\right\|}=0 \quad \text { in } E_{\text {rot }} \text {. }
$$

Iteration equations of the form (3.3), (3,4) are treated extensively in [6, Appendix B]; cf. also [12]. It is shown in [6, Corollary B.3 and Theorem B.9] that there exist

$$
\lambda^{ \pm}:=\lim _{n \rightarrow \pm \infty}\left\|v_{n}\right\|^{1 / n}
$$


and that these numbers are eigenvalues of $d S_{\text {rot }}(e)$ provided they are positive. It is obvious that $\lambda^{-}>0$, even $\lambda^{-} \geqq 1$, since $v_{n} \rightarrow 0$ as $n \rightarrow-\infty$. To show that $\lambda^{+}>0$, one can use the arguments of [6, proof of Theorem 3.1] where a similar property is shown for equations without singularity. We remark that the Lax theorem used in [6] applies in our case since the eigenvalues $\mu_{n}$ of the singular Sturm-Liouville problem

$$
\begin{gathered}
-v_{r r}-\frac{N-1}{r} v_{r}=\mu v \quad \text { in }(0,1), \\
v_{r}(0)=v(1)=0,
\end{gathered}
$$

satisfy $\mu_{n+1}-\mu_{n} \rightarrow \infty(n \rightarrow \infty)(c f .[23])$. Moreover, it is proved in [6] that the limits

$$
\varphi^{ \pm}:=\lim _{n \rightarrow+\infty} \frac{v_{n}}{\left\|v_{n}\right\|}
$$

exist and are eigenfunctions: $d S_{\text {rot }}(e) \varphi^{ \pm}=\lambda^{ \pm} \varphi^{ \pm}$. Using this and the inequalities $\lambda^{+} \leqq 1 \leqq \lambda^{-}$(which hold because $\left\|v_{n}\right\| \rightarrow 0$ as $n \rightarrow \pm \infty$ ), we obtain, by Proposition 3.2(C), that $\zeta\left(\varphi^{-}\right) \leqq \zeta\left(\varphi^{+}\right)$. Since $\varphi^{ \pm}$have only simple zeros in $[0,1]$, convergence in $C^{1}([0,1])$ gives

$$
\zeta\left(v_{-n}\right)=\zeta\left(\varphi^{-}\right) \leqq \zeta\left(\varphi^{+}\right)=\zeta\left(v_{n}\right)
$$

for large (positive) $n$. On the other hand $\zeta(v(\cdot, t))$ is nonincreasing in $t$. It follows that $\zeta(v(\cdot, t))$ is constant in $t \in \mathbb{R}$, and hence $v(\cdot, t)$ has only simple zeros in $r \in[0,1]$. Thus $v(0, t)$ is either negative or positive for all $t \in \mathbb{R}$, which implies that $\left(u_{n}(0)\right)_{n \in \mathbb{Z}}$ is a strictly monotone sequence. We infer that $\left(u_{n}\right)_{n \in \mathbb{Z}}$ cannot be a homoclinic orbit.

Step (iii). It remains to show

CLAIM 3.8. $K$ consists of just one element.

Proof. We first aim to find an asymptotically stable fixed point of $h$. Let $e \in K$ be a fixed point of the homeomorphism $h: K \rightarrow K$. We associate to $e$ the stable set $W^{s}(e):=\left\{y \in K: h^{n}(y) \rightarrow e\right.$ as $\left.u \rightarrow \infty\right\}$ and the unstable set $W^{u}(e):=\left\{y \in K: h^{-n}(y) \rightarrow e\right.$ as $n \rightarrow \infty\}$. Using the facts that there are only finitely many fixed points in $K$, that we have convergence of orbits by Proposition 3.2(B), and that we have ruled out cycles and homoclinic orbits, it is readily seen that at least one fixed point, say $\hat{e}$, has trivial unstable set $W^{u}(\hat{e})=\{\hat{e}\}$. We are going to apply [20, Lemma 2.9] to the homeomorphism $h$ in the compact metric space $K$ and the set $P:=\{\hat{e}\}$. For that we need to find a neighbourhood $Q$ of $\hat{e}$ in $K$ such that

$$
A:=\bigcap_{n \geq 0} h^{n}(Q)=\{\hat{e}\}
$$

This property holds if $Q$ is a compact neighbourhood of $\hat{e}$ in $K$, not containing any other fixed point of $h$. Indeed, note that $A \subset Q$ is negatively invariant under $h$, and assume there exists $z \in A, z \neq \hat{e}$. Then the negative semiorbit $\left(h^{-n}(z)\right)_{n \in \mathbb{N}}$ in $A$ converges, by Proposition 3.2(B), to a fixed point of $h$ in $Q$, thus to $\hat{e}$. This means that $z \in W^{n}(\hat{e})$, a contradiction.

Now [20, Lemma 2.9] implies the existence of a compact neighbourhood $\mathscr{U}$ of $\hat{e}$ in $K$, contained in $Q$, which is mapped into its interior by $h$. We may write $\mathscr{U}=$ 
$V \cap K$, where $V$ is a closed neighbourhood of $\hat{e}$ in the whole space $E$, with int $(\mathscr{U})=$ int $(V) \cap K$. Assume, for the sake of contradiction, that $K=\omega\left(u_{0}\right) \neq\{\hat{e}\}$. We find a sequence $n_{k} \geqq k, k \in \mathbb{N}$ such that $u_{n_{k}}:=S^{n_{k}}\left(u_{0}\right) \in$ int $(V)$ but $u_{n_{k}+1} \notin V$. Existence of such $n_{k}$ follows from the fact that $K=\omega\left(u_{0}\right)$ is not contained in $V \cap K=\mathscr{U} \subset Q$ (for otherwise, since $Q \subset K$ contains neither a cycle nor a fixed point different from $\hat{e}$, Proposition 3.2(B) would imply $K=\{\hat{e}\}$, a contradiction). Thus, for a subsequence, $u_{n_{\mathrm{k}}} \rightarrow y \in V \cap K=\mathscr{U}$, while $u_{n_{\mathrm{k}}+1} \rightarrow z=S(y)=h(y) \notin$ int $(V)$. Since $V \supset \mathscr{U}$, we found a point $y \in \mathscr{U}$ which is not mapped into int $(\mathscr{U})$ by $h$, a contradiction to the defining property of $\mathscr{U}$.

Thus $\omega\left(u_{0}\right)=\{\hat{e}\}$, which proves Theorem 3.1.

\section{Sufficient conditions for convergence}

We use the notation of the previous section. We first give two conditions under which the set

$$
\mathrm{Fix}^{+}(S):=\left\{u_{0} \in E: u\left(\cdot, t ; u_{0}\right) \geqq 0 \text { and } u\left(\cdot, t+T ; u_{0}\right)=u\left(\cdot, t ; u_{0}\right) \text { for } t \geqq 0\right\}
$$

is discrete. We know by Corollary 2.9 that $\mathrm{Fix}^{+}(S) \subset \mathrm{Fix}\left(S_{\text {rot }}\right)$, the set of fixed points of $S_{\text {rot }}$. First we discuss discreteness of Fix $\left(S_{\text {rot }}\right)$.

In view of the results on generic hyperbolicity in the autonomous case [3], one tends to believe that, generically with respect to $f(u, t)$ in an appropriate space, all fixed points of $S_{\text {rot }}$ are hyperbolic, hence isolated. However, for nonautonomous periodic equations, such a result is not available even in one space dimension, and does not seem to be an easy one. On the other hand, it can be proved without major difficulties that discreteness of Fix $\left(S_{\text {rot }}\right)$ is a generic property of $f(u, t)$. (We are not going to prove this here. But the proof uses the simplicity of eigenvalues of the linearisation of $\left(*_{\text {rot }}\right)$ and arguments similar to those in the proof of Lemma 4.1 below.)

We now consider the case of analytic $f$. Our sufficient conditions for property (P) to hold are based on the following lemma:

LEMMA 4.1. Let $f$ be analytic, and assume $e_{0}$ is a fixed point of $S_{\mathrm{rot}}$ which is not isolated. Then $e_{0}$ lies on a curve $\gamma$ of fixed points, and the value of $e_{0}(0)$ is in the interior of the interval $\{e(0): e \in \gamma\}$.

Proof. Consider the analytic operator $z \mapsto z-S_{\text {rot }}(z)$ on $E_{\text {rot }}$. As a compact perturbation of the identity, it is a Fredholm operator of index 0 . Since $e_{0}$ is not isolated, by the inverse function theorem the linearisation $I-d S_{\mathrm{rot}}(e)$ has a nontrivial kernel. By Proposition $3.2(\mathrm{C})$, the kernel is spanned by an eigenfunction $v$ having only simple zeros in $[0,1]$. We now apply the standard Lyapunov-Schmidt reduction [8] to obtain that there exists a neighbourhood $U$ of $e_{0}$ in $E_{\text {rot }}$, an $\varepsilon_{0}>0$, and analytic functions $\xi:\left(-\varepsilon_{0}, \varepsilon_{0}\right) \rightarrow E_{\text {rot }}$, respectively $\eta:\left(-\varepsilon_{0}, \varepsilon_{0}\right) \rightarrow \mathbb{R}$, such that

$$
\left\{z \in \mathscr{U}: z-S_{\text {rot }}(z)=0\right\}=\left\{s v+\xi(s): s \in\left(-\varepsilon_{0}, \varepsilon_{0}\right), \eta(s)=0\right\},
$$

with $\xi(0)=e_{0}, \dot{\xi}(0)=0$. Since $s=0$ is not an isolated zero of the analytic function $\eta$ (because $\xi(0)=e_{0}$ is not an isolated fixed point), we have $\eta \equiv 0$. We infer that the fixed point set in $\mathscr{U}$ is given by the analytic curve $\gamma=\left\{s v+\xi(s): s \in\left(-\varepsilon_{0}, \varepsilon_{0}\right)\right\}$.

We now look at the values of the solutions at $r=0, m(s):=s v(0)+\xi(s)(0)$. Since $m^{\prime}(0)=v(0) \neq 0$ (since $v$ has only simple zeros), the second claim also follows. 
We list now two cases in which property $(\mathrm{P})$ holds. Let $\lambda_{D}>0$ denote the principal eigenvalue of $-\Delta$ on $\Omega=\mathbb{B}(0)$, subject to Dirichlet boundary conditions.

Proposition 4.2. Let $f(u, t)$ be analytic, T-periodic in t, and satisfy

(F3) there exist smooth T-periodic functions $m(t), n(t)$ and constants $c \geqq 0 \geqq d$ such that $d+n(t) u \leqq f(u, t) \leqq c+m(t) u$ for all $u$ and $t$.

Assume

$$
\frac{1}{T} \int_{0}^{T} m(t) d t<\lambda_{D}
$$

and the same inequality with $m$ replaced by $n$. Then property $(\mathrm{P})$ holds.

Proof. We first claim that the set of $T$-periodic solutions of $(*)$ is $L^{\infty}$-bounded on $\bar{Q}_{T}:=\bar{\Omega} \times[0, \mathrm{~T}]$. Indeed, hypothesis (4.1) implies that the principal eigenvalue $\mu_{0}(m)$ of the periodic-parabolic eigenvalue problem

$$
\begin{cases}\partial_{t} \varphi-\Delta \varphi-m(t) \varphi=\mu_{0}(m) \varphi & \text { in } \Omega \times \mathbb{R}, \\ \varphi=0 & \text { on } \partial \Omega \times \mathbb{R}, \\ \varphi \text { is } T \text {-periodic in } t, & \end{cases}
$$

which can be easily calculated as $\mu_{D}(m)=\lambda_{0}-(1 / T) \int_{0}^{T} m(t) d t$, is positive [14, Lemma 15.3]. Let $u$ be $T$-periodic and satisfy $(*)$ :

$$
\partial_{t} u-\Delta u=f(u, t) \leqq c+m(t) u,
$$

and let $\psi \gg 0$ be the unique $T$-periodic solution of

$$
\begin{cases}\partial_{t} \psi-\Delta \psi-m(t) \psi=c & \text { in } \Omega \times \mathbb{R}, \\ \psi=0 & \text { on } \partial \Omega \times \mathbb{R} .\end{cases}
$$

(cf. [14, Theorem 16.6]). For any $\delta>1, \delta \psi-u$ is a periodic function satisfying

$$
\partial_{t}(\delta \psi-u)-\Delta(\delta \psi-u)-m(t)(\delta \psi-u) \geqq(\delta-1) c>0,
$$

hence $\delta \psi-u \gg 0$ (cf. [14, Remark 16.7]). It follows that $u \leqq \psi$. In an analogous way one finds a bound from below. This proves the $L^{\infty}$-boundedness.

We next prove that $\mathscr{G}:=$ Fix $\left(S_{\text {rot }}\right)$ is discrete, in particular, $(\mathrm{P})$ holds. From the claim just proved, it follows that $\mathscr{G}$ is relatively compact in $E_{\text {rot }}$. Assume $\mathscr{G}$ is not discrete. Then the subset $\mathscr{H}$ of $\mathscr{G}$ consisting of all nonisolated fixed points is nonempty and compact in $E_{\text {rot }}$. Let $\eta:=\max \{e(0): e \in \mathscr{H}\}$. Then $\eta=e_{0}(0)$ for some $e_{0} \in \mathscr{H}$, contradicting the second assertion of Lemma 4.1.

Proposition 4.3. Let $f(u, t)$ be analytic and T-periodic in t. Assume

(F4) $f(0, \cdot) \geqq 0$ but $f(0, \cdot) \not \equiv$.

Then property $(\mathrm{P})$ holds.

Proof. First note that, by (F4) and the maximum principle, any non-negative initial condition gives rise to a non-negative solution of $(*)$. Let $F$ be the set of nonisolated non-negative fixed points of $S$ (hence of $S_{\text {rot }}$ ). Assume, for contradiction, that the set $\mathscr{F}$ (of nonisolated fixed points) defined above is nonempty, and let $e_{1} \in \mathscr{F}$. Let the 
constant $M$ be such that $e_{1}(0)<M$, and define

$$
\mathscr{F}_{M}:=\{e \in \mathscr{F}: e(0) \leqq M\} .
$$

Since, by Corollary $2.9, e(0)=\max e$ for any $e \in \mathscr{F}, \mathscr{F}_{M}$ is bounded in $L^{\infty}$, hence compact in $E_{\text {rot }}$. Of course $\mathscr{F}_{M} \neq \varnothing$, since $e_{1} \in \mathscr{F}_{M}$. Let $\eta:=\min \left\{e(0): e \in \mathscr{F}_{M}\right\}$. There exists $e_{0} \in \mathscr{F}_{M}$ such that $e_{0}(0)=\eta$. By $(\mathrm{F} 4), 0$ is a strict subsolution for the periodic Dirichlet problem for $\partial_{t} u-\Delta u=f(u, t)$. It follows by the maximum principle that the periodic solution $u$ associated to the initial value $u(\cdot, 0)=e_{0}$ satisfies $u(x, t)>0$ for $x \in \Omega, \partial_{v} u(x, t)<0$ for $x \in \partial \Omega$, for all $t$. Thus also the nearby periodic solutions to initial conditions on the curve $\gamma$ (given by Lemma 4.1) are positive. This contradicts the choice of $e_{0}$.

By Theorem 3.1 and Proposition 4.3, non-negative bounded solutions of (*) converge to $T$-periodic solutions provided the analytic nonlinearity $f$ satisfies (F4). This may now be strengthened to the next theorem:

TheOREM 3.1'. Let $f(u, t)$ be analytic and T-periodic in t, and assume $\left(\mathrm{F} 4^{\prime}\right) f(0, \cdot) \geqq 0$.

Then all bounded non-negative solutions of $(*)$ converge (in E) to T-periodic solutions of $(*)$.

Proof. We only have to consider the case where $f(0, \cdot)=0$. Clearly $u=0$ is then the periodic solution of $(*)$. If we assume the existence of nonisolated positive periodic solutions for which the curve $\gamma$ (given by Lemma 4.1) does not contain 0 , we reach a contradiction as in the proof of Proposition 4.3 (considering in $\mathscr{F}_{M}$ only elements on that curve). Hence there can only be nonisolated fixed points of $S_{\text {rot }}$ on a curve $\gamma_{0}$ through 0 . Since nontrivial non-negative periodic solutions are indeed positive by $\left(\mathrm{F} 4^{\prime}\right)$, we infer that at $e_{0}=0$ the vector $v$ giving the tangent direction of the curve $\gamma_{0}$ (i.e. giving rise to a periodic eigenfunction of the problem linearised at 0 ) is nonnegative, hence lies in the interior of the positive cone of $E_{\text {rot }}$ by the properties of the principal eigenfunctions. By the maximum principle and a continuity argument, this remains true for all non-negative elements on the curve $\gamma_{0}$. We infer that the positive part of $\gamma_{0}$ is totally ordered. It is moreover unbounded above (in $L^{\infty}$ ), since we could otherwise reach a contradiction as in the proof of Proposition 4.2. Clearly all elements on the positive part of $\gamma_{0}$ are order-stable, hence stable by [14, Lemma 4.3]. Moreover, there is no other (i.e. isolated) non-negative fixed point. Indeed, such an element $\bar{e}$ would lie in the interior of the positive cone of $E_{\mathrm{rot}}$. Choose the member of $\gamma_{0}$ which touches $\bar{e}$ from below. This contradicts the fact that $S_{\text {rot }}$ is strongly order-preserving: $e=S_{\text {rot }}(e) \ll S_{\text {rot }}(\bar{e})=\bar{e}$ (in the sense of the order cone in $E_{\text {rot }}$ ). Thus all non-negative fixed points of $S$ are stable. Convergence of all non-negative orbits follows now by [14, Theorem 3.3].

We remark that this proof is strongly related to that of [14, Proposition 25.1] on equations of Fisher's type.

\section{References}

1 S. B. Angenent. Solutions of the $1-d$ porous medium equation are determined by their free boundary J. London Math. Soc. 42 (1990), 339-353. 
2 H. Berestycki and L. Nirenberg. On the method of moving planes and the sliding method (preprint, École Normale Supérieure, 1991).

3 P. Brunovský and P. Poláčik. Generic hyperbolicity for reaction-diffusion equations on symmetric domains. J. Appl. Math. Phys. (ZAMP) 38 (1987), 172-183.

4 P. Brunovskỳ, P. Poláčik and B. Sandstede. Convergence in general periodic parabolic equations in one space dimension. Nonlinear Anal. 18 (1992), 209-215.

5 A. Castro and R. Shivaji. Nonnegative solutions to a semilinear Dirichlet problem in a ball are positive and radially symmetric. Comm. Partial Differential Equations 14 (1989), 1091-1100.

6 M. Chen, X.-Y. Chen and J. K. Hale. Structural stability for time-periodic one-dimensional parabolic equations. J. Differential Equations 96 (1992), 355-418.

7 X.-Y. Chen and H. Matano. Convergence, asymptotic periodicity, and finite-point blow up in onedimensional semilinear heat equations. J. Differential Equations 78 (1989), 160-190.

8 S. N. Chow and J. K. Hale. Methods of Bifurcation Theory (New York: Springer, 1982).

9 E. N. Dancer and P. Hess. Stable subharmonic solutions in periodic reaction diffusion equations. J. Differential Equations (to appear).

10 E. N. Dancer and P. Hess. The symmetry of positive solutions of periodic-parabolic problems (preprint).

11 B. Gidas, W. Ni and L. Nirenberg. Symmetry and related properties by the maximum principle. Comm. Math. Phys. 68 (1979), 209-243.

12 D. Henry. Some infinite-dimensional Morse-Smale systems defined by parabolic partial differential equations. J. Differential Equations 59 (1985), 165-205.

13 P. Hess. Asymptotics in semilinear periodic diffusion equations with Dirichlet or Robin boundary conditions. Arch. Rational Mech. Anal. 116 (1991), 91-99.

14 P. Hess. Periodic-Parabolic Boundary Value Problems and Positivity, Pitman Research Notes in Mathematics 247 (Harlow: Longman, 1991).

15 P. Hess and P. Poláčik. Boundedness of prime periods of stable cycles and convergence to fixed points in discrete monotone dynamical systems. SIAM J. Math. Anal. (to appear).

16 P. Hess and $\mathrm{H}$. Weinberger. Convergence to spatial-temporal clines in the Fisher equation with time-periodic fitnesses. J. Math. Biol. 28 (1990), 83-98.

17 P. Poláčik and I. Terešćák. Convergence to cycles as a typical asymptotic behaviour in smooth strongly monotone discrete-time dynamical systems. Arch. Rational Mech. Anal. 116 (1991), 339-360.

18 P. Poláčik and I. Tereščák. Exponential separation and invariant bundles for maps in ordered Banach spaces with applications to parabolic equations. J. Dynamical Differential Equations 5 (1993) 279-303.

19 J. Serrin. A symmetry problem in potential theory. Arch. Rational Mech. Anal. 43 (1971), 304-318.

20 M. Shub. Global Stability of Dynamic Systems (New York: Springer, 1987).

21 P. Takáč. Linearly stable subharmonic orbits in strongly monotone time-periodic dynamical systems. Proc. Amer. Math. Soc. 115 (1992), 691-698

22 P. Takáč. A construction of stable subharmonic orbits in monotonic time-periodic dynamical systems Mh. Math. 115 (1993), 215-244.

23 G. N. Watson, $A$ Treatise on the Theory of Bessel Functions (Cambridge: University Press 1944).

(Issued 16 June 1994) 